\title{
Further Experience with Indomethacin in the Treatment of Rheumatic Disorders
}

\author{
M. THOMPSON,* M.D., M.R.C.P. ; J. S. PERCY,*† M.B., B.S., M.R.C.P.BD.
}

Brit. med. F., 1966, 1, 80-83

Indomethacin (Indocid) is an indole-3-acetic acid derivative, which was found to have powerful anti-inflammatory and analgesic properties, and which has been under clinical investigation since October 1962. Several controlled trials demonstrated that it had some value in the treatment of rheumatic disorders, being significantly more effective than placebos in the treatment of both rheumatoid arthritis (Wanka, Jones, Wood, and Dixon, 1964) and osteoarthrosis of the hip (Wanka and Dixon, 1964). When indomethacin was compared with phenylbutazone in a controlled trial in rheumatoid arthritis (Percy, Stephenson, and Thompson, 1964) no significant difference was noted either in terms of relief of pain or by improvement in grip-strength, but side-effects were commoner with indomethacin. All these early studies were undertaken with indomethacin in tablet form, and it was found that this preparation had disadvantages owing to variable dissolution rates of the tablets and erratic absorption from the gastro-intestinal tract. The use of capsules, available since September 1963, overcame these difficulties, and subsequent clinical experience indicated that a commensurate clinical effect could be obtained with a lower dosage of indomethacin in capsule form than when it was administered as tablets (Thompson, 1964).

Experience with other drugs used in the treatment of chronic rheumatic disorders, notably with the predni-steroids, has shown that potency of a drug does not establish its place as the therapeutic agent of choice. Controlled clinical trials of short duration serve to estimate the immediate value of a drug and any early side-effects, but it is only by means of long-continued clinical experience that answers may be provided to such questions as the frequency, severity, and nature of adverse sideeffects, optimum dosage levels, and the indications for or against the use of the drug. As indomethacin became available for use in general practice in March 1965 we are recording our experience of a group of 137 patients treated with 25-mg. capsules during the 82 weeks from September 1963 to April 1965.

The diagnoses of the 137 patients are listed in Table I, the majority being rheumatoid arthritis or osteoarthrosis. All patients were treated in the out-patient department and were

TABLE I.-Composition of the Group of 137 Patients

\begin{tabular}{|c|c|c|c|c|c|}
\hline Rheumatoid arthritis & .. & 70 & Psoriatic arthritis & . & 2 \\
\hline Osteoarthrosis & .. & 50 & Polymyalgia rheumatica & . & 2 \\
\hline Ankylosing spondylitis & . & 4 & Systemic sclerosis & . & 1 \\
\hline $\begin{array}{ll}\text { Gout } & \\
\text { Cervical spondylosis } & \text {.. }\end{array}$ & $\because$ & $\begin{array}{l}3 \\
3\end{array}$ & $\begin{array}{l}\text { Shoulder-hand syndrome } \\
\text { Reiter's syndrome }\end{array}$ & $\cdots$ & 1 \\
\hline & & & & & \\
\hline
\end{tabular}

seen at intervals of one to eight weeks according to the severity of their symptoms, the duration of their treatment, and the presence of side-effects. Regular urine analysis, blood counts, and liver-function tests were done in all patients receiving treatment for one month or longer. Indomethacin was administered in 25-mg. capsules taken after meals with an adequate amount of fluid. Previous experience had shown that a progressive dosage regime reduced the incidence of early side-effects, so

\footnotetext{
- Department of Physical Medicine, Royal Victoria Infirmary, Newcastle upon Tyne.

† Registrar, Professorial Medical Unit, Royal Victoria Infirmary. Newcastle upon Tyne.
}

treatment was inaugurated with one capsule daily after breakfast for three to seven days, increasing thereafter to two capsules taken at 12-hourly intervals, and later to three or more capsules daily, in divided dosage. When indomethacin was given to patients currently receiving phenylbutazone, the latter preparation was discontinued. Patients on aspirin or predni-steroids maintained dosage of these preparations at the same level initially, then reduced or stopped them if the response to indomethacin was satisfactory. The results of our experience are summarized under the headings of rheumatoid arthritis, osteoarthrosis, miscellaneous rheumatic disorders, and side-effects.

\section{Rheumatoid Arthritis}

Seventy patients suffering from classical or definite rheumatoid arthritis, according to the American Rheumatism Association (1959) criteria, received indomethacin for periods ranging from 1 to 82 weeks, the mean duration being 33 weeks. The average daily maintenance dosage was $75 \mathrm{mg}$., with a range from 25 to $150 \mathrm{mg}$. The results of treatment are shown in Table II. Three patients developed intercurrent illnesses un-

TABLE II.-Comparison of Results of Treatment in Rheumatoid

\begin{tabular}{|c|c|c|}
\hline Outcome & $\begin{array}{l}\text { Rheumatoid Arthritis } \\
\text { (67 Patients) }\end{array}$ & $\begin{array}{l}\text { Osteoarthrosis } \\
\text { (49 Patients) }\end{array}$ \\
\hline $\begin{array}{l}\text { Continued therapy } \\
\text { Stopped; full remission } \\
\text { "y lack of benefit } \\
\text { side-effects }\end{array}$ & $\begin{array}{l}27(40 \%)\}(44 \%) * \\
3(4 \%) \\
22(33 \%) \\
15(22 \%)\end{array}$ & $\begin{array}{l}31(63 \%)) \\
3(6 \%)\}(69 \%) \\
11(23 \%) \\
4(8 \%)\end{array}$ \\
\hline $\begin{array}{c}\text { Patients' estimate } \\
\text { of benefit }\end{array}$ & \begin{tabular}{lr|}
\multicolumn{3}{c|}{ (70 patients) } \\
Satisfactory & $18(26 \%)$ \\
Fairly good & $28(40 \%)$ \\
Slight or absent $24(34 \%)$
\end{tabular} & \begin{tabular}{ll}
\multicolumn{2}{c}{ (50 patients) } \\
Satisfactory $28(55 \%)$ \\
Fairly good $14(29 \%)$ \\
Slight or absent $8(16 \%)$
\end{tabular} \\
\hline
\end{tabular}

Note: Three rheumatoid patients who stopped treatment owing to intercurrent illness and one osteoarthrotic patient lost to follow-up were not included in the assessment of outcome.

* Indicates significant differences between the two columns.

related to the rheumatoid condition or to therapy, but indomethacin was withdrawn to avoid the complicating factor of a new drug in their management, so these patients were not included in the assessment of outcome. Of the remaining 67 patients $27(40 \%)$ continued the treatment, and to this number should be added the three patients who obtained a complete remission, giving a total of $30(44 \%)$ for whom treatment may be classed as completely or reasonably successful.

The patient's estimate of benefit was based upon several factors, notably relief of pain, reduction of morning stiffness, and comparison with previous therapy when this had been replaced by indomethacin. The influence of side-effects was exclude 1 from this assessment. By these criteria $18(26 \%)$ felt that indomethacin was the most effective drug they had received, $28(40 \%)$ considered it to be reasonably effective, and $24(34 \%)$ found it to be relatively or absolutely ineffective.

Side-effects occurred in $26(37 \%)$ patients (Table III), and necessitated withdrawal of treatment in 22 . Inadequate clinical effect was the reason for stopping treatment in a further 15 $(22 \%)$, making a total of $37(55 \%)$ who stopped taking the drug because it was unsatisfactory. These two factors of side- 
effects and poor clinical response are to some extent interdependent, as lack of benefit led in eight cases to increasing the dosage to a point where satisfactory analgesic effect was obtained only by the development of unacceptable side-effects.

\section{Osteoarthrosis}

Fifty patients suffering from osteoarthrosis were treated for periods ranging from 2 to 82 weeks, the mean duration being 28 weeks. The average daily maintenance dosage was $75 \mathrm{mg}$., the range being 25 to $125 \mathrm{mg}$. The results of treatment are shown in Table II. Only one patient was lost to follow-up through failure to attend. Of the remaining 49 patients 31 continued with treatment, and to these may be added the three patients who obtained a complete remission of symptoms, making a total of $34(69 \%)$ in whom the outcome was regarded as completely or reasonably successful.

The patients' estimate of benefit was based upon the factors of pain relief, reduction of stiffness, improvement in functional capacity, and comparison with previous therapy. By these criteria $28(56 \%)$ patients thought that indomethacin was satisfactory, $14(28 \%)$ found it to be fairly good, and only $8(16 \%)$ considered it to be relatively or absolutely ineffective.

Side-effects occurred in $14(28 \%)$ patients and were responsible for the withdrawal of the drug in 11 . The incidence of side-effects was lower than in the rheumatoid group, but not significantly so. The number of patients withdrawn because of inadequate effect was much lower in the osteoarthrotic patients $(8 \%)$ than in those suffering from rheumatoid disease $(22 \%)$, and this difference was statistically significant $\left(\chi^{2}=4, P<0.05\right)$. When the outcome of therapy was compared there was a significantly favourable result for the osteoarthrotic group in comparison with the rheumatoid patients $\left(\chi^{2}=6, P<0.02\right)$. Furthermore, when the patient's estimate of benefit was considered, the numbers claiming satisfactory benefit were significantly higher in the osteoarthrotic group $\left(\chi^{2}=10, \mathrm{P}<0.005\right)$.

\section{Miscellaneous Group}

The composition of this group of 17 patients is shown in Table I, and these patients received treatment for periods ranging from 1 to 55 weeks, the mean being 13 weeks. The average dosage was $87 \mathrm{mg}$. daily, the range being 50 to $150 \mathrm{mg}$. Seven patients experienced side-effects compelling withdrawal of the drug in six cases, and in a further two it was withdrawn because of inadequate benefit. Two patients stopped taking indomethacin when they left the district, and in three the drug was stopped because of satisfactory remission of symptoms. Four patients continued to take indomethacin. The numbers involved are too small to permit detailed analysis of the results in the group as a whole or in the various diagnostic subgroups, but some observations on the outcome are presented.

Of the two patients suffering from psoriatic arthritis, one improved and continued with treatment but the other developed side-effects within two weeks and had to stop. A similar response was observed with the two patients suffering from polymyalgia rheumatica, one of whom developed side-effects, while the other found indomethacin to be effective and was able to stop steroid therapy. None of the three patients with cervical spondylosis obtained striking benefit, but all had complicating illnesses and had been refractory to previous drugs and physiotherapy.

Four patients suffering from ankylosing spondylitis were treated and three had to discontinue because of side-effects, the therapeutic response being rated as satisfactory, good, and slight respectively. The fourth patient continued treatment with satisfactory effect.
A strikingly favourable response was obtained in the three patients treated for gout: acute symptoms were rapidly brought under control in the two who received daily maintenance doses of 75 and $125 \mathrm{mg}$. respectively, and were then able to stop treatment in complete remission; the thind patient with chronic gout was experiencing intermittent painful episodes, and these have remained suppressed while treatmert has continued with a maintenance dosage of $50 \mathrm{mg}$. daily.

The patient suffering from shoulder-hand syndrome following coronary thrombosis obtained fairly good relief of symptoms with a maintenance dosage of $100 \mathrm{mg}$. daily given for seven weeks, and the patient with Reiter's syndrome continued therapy with good effect on a dosage of $75 \mathrm{mg}$. daily. The last patient in this group, who had a painful arthropathy complicating systemic sclerosis, developed side-effects on minimal dosage of indomethacin.

\section{Side-effects}

The number and the nature of the side-effects encountered among 137 patients are listed in Table III. A total of 70 symptoms were recorded in $47(34 \%)$ patients, and compelled withdrawal of therapy in $39(28 \%)$ cases. Though the incidence was lower in the osteoarthrotic group, there were no significant differences between the three groups in either the incidence or character of the symptoms. There were no deaths from any cause in any patient receiving indomethacin during the entire period of the trial. Furthermore, no significant or sustained adverse effects were noted on the haematopoietic, hepatic, or renal organs as judged by repeated laboratory investigations.

TABLE III.-Side-effects Attributed to Indomethacin

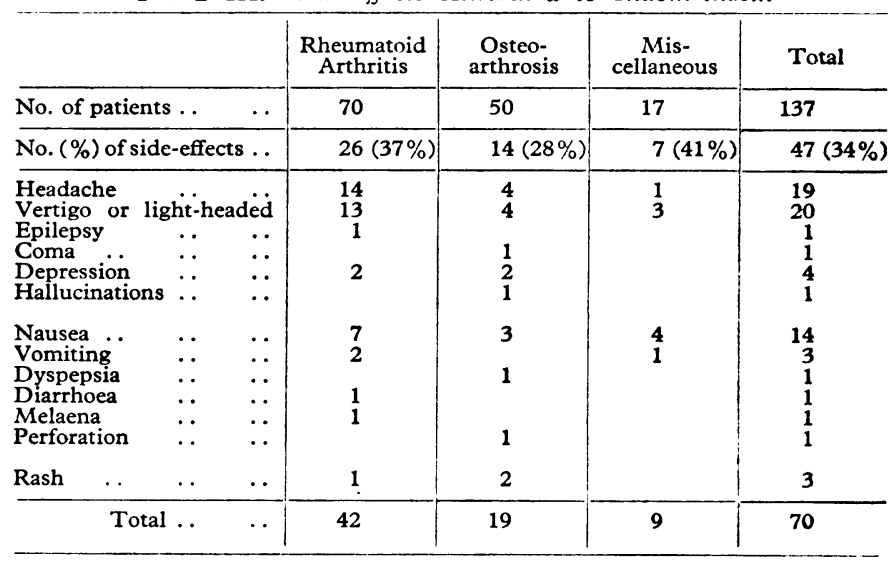

The side-effects could be classified in three main groups, which, in order of frequency, were neurological, gastrointestinal, and dermatological. The commonest symptom was headache, which was variable in its severity, character, and distribution and was not associated with any apparent vascular changes. Most frequently the headache was located centrally in the skull or at the vertex and occurred on first waking or shortly after the first capsule of the day had been taken. In the majority of cases the headache was associated with vertigo, light-headedness, or a feeling of slight confusion. In the most severe cases there was associated nausea, malaise, and vomiting, variously described as motion-sickness or moderate inebriation. When the headache was slight it was possible to avoid it by temporary reduction of the dosage, and five patients were able to continue treatment after this had been done. Seven patients experienced major neurological disturbances, four becoming severely depressed and three developing coma, epilepsy, and hallucinations respectively. The four women who developed depressive symptoms did so after long periods on treatment, the average being 37 weeks before symptoms developed. In the 
other three cases the maintenance dosage had been $100 \mathrm{mg}$. daily or above.

Depressive symptoms developed insidiously in all four patients who, lacking insight into the cause of their trouble, continued to take the capsules. One patient stated that she had contemplated suicide. The depression cleared rapidly when the capsules were stopped. Two of these patients later resumed indomethacin therapy in lower dosage, without any recurrence of depression during eight weeks' therapy. This was justified on the grounds that indomethacin had given the most benefit in the treatment of their arthritic symptoms and that both these patients and their relatives were alerted to the necessity for drug withdrawal should any neuro-psychiatric symptoms recur. The patient who became comatose was a 43-year-old man who had taken indomethacin for two weeks, had reached the $75 \mathrm{mg}$. daily dosage level, and was not taking any other drugs. $\mathrm{He}$ became comatose within half an hour of feeling confused, and three hours after taking the first capsule of that day, and may have taken an extra capsule. The coma began to clear in three hours and he remained stuporous until the following day. $\mathrm{He}$ was admitted to hospital, where no localizing signs were found, and investigations failed to reveal any cause, other than indomethacin, for his condition.

One patient, a man aged 52, had three epileptiform seizures while taking indomethacin. The first was a Jacksonian attack involving the right arm, and this occurred during the second week of therapy. The second attack began with a Jacksonian fit but in addition involved some transient paresis of both legs. The third seizure occurred during the third week of treatment, when the dosage was $75 \mathrm{mg}$. daily, and started as a Jacksonian attack but progressed to a grand-mal fit with brief loss of consciousness. Subsequent neurological investigation and electroencephalographic examinations were negative and the patient has had no further seizures since stopping indomethacin one year ago.

The patient who experienced visual and auditory hallucinations was a 72 -year-old woman who had been taking $50 \mathrm{mg}$. of indomethacin daily for 31 weeks with good effect for an osteoarthrotic hip. She saw and conversed with various friendly ghosts whose visitations were becoming more frequent until the capsules were stopped. This patient also had atherosclerosis and was receiving methyldopa in the treatment of hypertension, but she has not had further hallucinations in the three-month period since indomethacin was stopped.

As most of the 14 patients complaining of nausea and two of the three cases of vomiting were associated with headache and vertigo, the incidence of side-effects originating in the gastro-intestinal tract was small. The only major incident was perforation of an acute duodenal ulcer in a 67-year-old woman who had taken $75 \mathrm{mg}$. of indomethacin daily for 14 weeks, and had no preceding history of dyspepsia. This patient later continued therapy with indomethacin by suppositories, $100 \mathrm{mg}$. daily, with relief of her hip pain.

One patient, who had a long previous history of peptic ulceration and had been unable to take other analgesics and anti-inflammatory drugs, took indomethacin tablets for 14 weeks and then capsules for 26 weeks before she had a slight melaena. As indomethacin gave such symptomatic relief she later tried suppositories but was unable to retain these.

Three patients developed a diffuse erythematous macular rash, associated with mild stomatitis in one case. In all cases the rash cleared within a day of discontinuing treatment. One of these patients resumed taking indomethacin on her own initiative but stopped it when the rash occurred within a few days. Possible, but not proved, side-effects included $(a)$ the occurrence of furunculosis in two men, which cleared while they continued with indomethacin; $(b)$ the aggravation of leg oedema in two women, both of whom were receiving steroid therapy, and $(c)$ the aggravation of pre-existing mild diabetes in a man.

\section{Discussion}

It is clear from reported clinical experience that indomethacin is a powerful compound, capable of giving relief of symptoms and reduction of inflammatory signs in several rheumatic disorders. The most striking reduction of inflammatory signs has been noted in gout and in acute articular exacerbations of rheumatoid disease. However, the greatest benefit has been obtained in osteoarthrosis, particularly when the hip-joints were involved. The results of treatment were significantly better in the osteoarthrotic than in the rheumatoid group when judged by the criteria of (a) fewer failures owing to lack of effect, $(b)$ numbers of patients able to continue with satisfactory relief of symptoms without experiencing side-effects, and (c) the patients' own estimates of benefit.

Of the total group of 137 patients 39 (28\%) had to stop treatment because of unacceptable side-effects and 21 (15\%) because of lack of benefit, while six patients stopped treatment for other reasons. Nine patients stopped because of satisfactory remission and 62 continued to take the drug, making $71(52 \%)$ in whom the outcome has been satisfactory. This proportion of success must be judged against the background of the previous history of these patients, as no patient was treated with indomethacin $a b$ initio but only when they presented a therapeutic problem. Thus all 70 rheumatoid patients had received aspirin, 35 had been given oral predni-steroid therapy, and 44 had been treated with one or more anti-inflammatory preparations-for example, phenylbutazone, gold salts, or chloroquine preparations. In the miscellaneous group of 17 patients 14 had received aspirin, 5 oral predni-steroids, and all 17 had taken other anti-inflammatory preparations, mainly phenylbutazone. In the group of 50 patients suffering from osteoarthrosis the previous therapy had comprised aspirin in 48 cases, oral prednisteroid therapy in 5, and other anti-inflammatory drugs in 40. In these circumstances the results obtained indicate that indomethacin has provided a most useful additional therapy in the management of osteoarthrosis, gout, and some patients suffering from rheumatoid arthritis. Although in our experience gout treated by capsules of indomethacin has been limited to three cases, the results obtained in these patients, supplemented by our experience with three patients treated by tablets, fully confirm the beneficial effects recorded by Hart and Boardman (1963), who noted the particular value of indomethacin in controlling acute gouty symptoms.

In general, the response to indomethacin could be predicted within the first few days of treatment, as those who were going to obtain substantial benefit often responded to small doses. Daily maintenance dosage of 50 or $75 \mathrm{mg}$. was usually adequate, occasional patients requiring $100 \mathrm{mg}$. or more. The absolute maximum dosage that was used was $150 \mathrm{mg}$. daily. The limiting factor was the development of undesirable effects, which became more frequent as dosage increased. The value of a slowly progressive dosage regime has already been demonstrated in the use of tablets in reducing the incidence of headache in the early stages of treatment (Percy et al., 1964). A further advantage of the initial low-dosage regime is that patients extremely intolerant of indomethacin will be detected early, so that though unacceptable side-effects occur the risk of major trouble is limited. Five patients in our series had troublesome side-effects of headache and vertigo while on the minimum dosage of $25 \mathrm{mg}$. daily and were compelled to stop treatment. This is a point of considerable importance, as the headache, vertigo, and "muzziness" so commonly encountered during indomethacin therapy may occur acutely and episodically, and patients should be warned of the dangers of traffic, either as pedestrians or drivers.

We found the clinical response to indomethacin to be similar in many respects to that seen with phenylbutazone, which has been used extensively in the treatment of generalized osteoarthrosis, osteoarthrosis of the hip, acute gout, rheumatoid 
arthritis, particularly with actively inflamed large joints, and ankylosing spondylitis. The fact that indomethacin is a rapidly effective, non-steroidal, anti-inflammatory agent, like phenylbutazone, makes it a useful alternative to predni-steroid therapy.

As with many other drugs used in the treatment of chronic rheumatic disorders, indomethacin causes undesired effects which are particularly apt to occur in the early stages of treatment. Fortunately most of these are trivial and clear rapidly after withdrawal of the drug. In view of the occurrence of severe neurological disturbance in seven of our cases we feel that it would be unwise to ignore and dangerous to suppress the headache and associated symptoms so commonly encountered. If these cannot be avoided by gradual induction or by subsequent reduction of dosage, they must be taken as an indication for stopping the drug. It would seem reasonable to insist that indomethacin should only be given with caution to any patient with a history of depressive illness. Active or recent peptic ulceration should be regarded as an absolute contraindication, and therapy should not be resumed in any patient who exhibits a rash during treatment.

\section{Summary}

Clinical experience with indomethacin during a period of 18 months in a series of 137 patients comprising 70 with rheumatoid arthritis, 50 with osteoarthrosis, and 17 with miscellaneous rheumatic disorders is recorded. A progressive dosage regime was used beginning with $25 \mathrm{mg}$. daily, and the average daily maintenance dosage was $75 \mathrm{mg}$.
Striking benefit was obtained in osteoarthrosis with a satisfactory outcome of treatment in $69 \%$ of cases, which was significantly better than the rheumatoid group where $44 \%$ were satisfactory. An excellent response was also seen in three patients suffering from acute gout.

A total of 70 side-effects occurred in 47 patients and compelled withdrawal of treatment in 39 ( $28 \%$ of the total group). The principal undesired symptoms were headache associated with vertigo, etc., but major neurological disturbance occurred in seven cases. All these symptoms subsided rapidly on withdrawal of therapy. There was one instance of slight melaena, one perforated ulcer, and three rashes developed during therapy.

Indomethacin is thought to be a useful addition to the range of drugs used in the management of certain rheumatic disorders, notably in osteoarthrosis, acute gout, rheumatoid arthritis, and spondylitis.

We wish to thank Dr. R. Hodgkinson, of Merck Sharp \& Dohme, Hoddesdon, Herts, for his co-operation and for supplies of indomethacin capsules. We also thank Drs. M. Hall, R. A. H. Morison, and R. A. Sutton for help in the care of the patients, and Miss $\mathbf{P}$. Port for secretarial assistance.

\section{REFERENCES}

American Rheumatism Association (1959). Ann. rheum. Dis., 18, 49. Hart, F. D., and Boardman, P. L. (1963). Brit. med. 7., 2, 965.

Percy, J. S., Stephenson, P., and Thompson, M. (1964). Ann. rheum. Dis., 23, 226

Thompson, M. (1964). Rhumatologie, 16, 439.

Wanka, J., and Dixon, A. St. J. (1964). Ann. rheum. Dis., 23, 288.

Iones, L. I., Wood. P. H. N., and Dixon, A. St. J.'(1964). Ibid., 23,218 .

\title{
Antibiotic Treatment of Epidemic Bronchiolitis-a Double-blind Trial
}

\author{
C. M. B. FIELD,* M.D., F.R.C.P.ED. ; J. H. CONNOLLY, † M.D., M.R.C.P.I. ; G. MURTAGH,* M.B., B.CH., B.A.O. \\ CLAIRE M. SLATTERY,* M.B., B.CH., B.o.A.; E. E. TURKINGTON, $\ddagger$ B.SC.
}

Brit. med. F., 1966, 1, 83-85

Epidemic bronchiolitis is a common and widespread disorder of infants in Great Britain (Heycock and Noble, 1962; Holzel, Parker, Patterson, Cartmel, White, Purdy, Thompson, and Tobin, 1965). It has been shown in the U.S.A. (Beem, Wright, Hamre, Egerer, and Oehme, 1960 ; Chanock, Kim, Vargosko, Deleva, Johnson, Cumming, and Parrot, 1961; McClelland, Hilleman, Hamparian, Ketler, Reilly, Cornfeld, and Stokes, 1961) and in Great Britain (Sandiford and Spencer, 1962 ; Holzel, Parker, Patterson, White, Thompson, and Tobin, 1963) that respiratory syncytial virus is the predominant cause of bronchiolitis in infancy. Previous studies in Northern Ireland in 1962 and 1963 have also shown this association (Connolly, Forsyth, Haire, Evans, and White, 1963).

There is not as yet general agreement on the therapy of bronchiolitis in infancy. Wide-range antibiotics are advocated by Holt, McIntosh, and Barnett (1962) and by Jolly (1964), though Shirkey (1964) does not consider them to be of value. In view of this difference of opinion and of their almost universal use in domiciliary practice it was decided to plan a trial in order to test the efficacy of one of the antibiotics. As tetracycline had been shown to cause abnormalities in the teeth

* Paediatric Department, Belfast City Hospital.

₹ Statistician, Queen's University, Belfast. of infants (Wallman and Hilton, 1962), ampicillin, which has been proved to be effective in certain respiratory disorders in children (Elliot, Stokes, and Maxwell, 1964), was chosen.

\section{Patients, Materials, and Methods}

An epidemic of bronchiolitis occurred in Belfast in November 1964. The criteria of selection for the trial were coryza, paroxysmal cough, expiratory wheeze, and increased resp'ratory rate. All patients except three had fine crepitations at the lung bases and were sufficiently distressed to be using the accessory muscles of respiration. The progress of each case was reported daily on a special chart which listed pulse rate, temperature, respiratory rate, the use of accessory muscles of respiration, expiratory wheeze, adventitious sounds in the chest, and the presence of cyanosis.

The treatment consisted of ampicillin or placebo $125 \mathrm{mg}$. six-hourly, all children received ephedrine $\frac{1}{4}$ gr. $(16 \mathrm{mg}$.) thrice daily, and most of them were nursed for a day or two in an oxygen tent with aerosol water vapour. One or other treatment was allocated to the children according to a randomized code. It was decided that patients who became dangerously ill owing to steady deterioration of their condition should be given an antibiotic and removed from the trial. This was a difficult 